\title{
SLUG: A Connectionist Architecture for Inferring the Structure of Finite-State Environments
}

\author{
MICHAEL C. MOZER \\ (MOZER@CS.COLORADO.EDU) \\ Department of Computer Science, and Institute of Congnitive Science, University of Colorado, Boulder, CO \\ $80309-0430$
}

JONATHAN BACHRACH

(BACHRACH@CS.UMASS.EDU)

Department of Computer and Information Science, University of Massachusetts, Amherst, MA 01003

\begin{abstract}
Consider a robot wandering around an unfamiliar environment, performing actions and sensing the resulting environmental states. The robot's task is to construct an internal model of its environment, a model that will allow it to predict the consequences of its actions and to determine what sequences of actions to take to reach particular goal states. Rivest and Schapire (1987a, 1987b; Schapire, 1988) have studied this problem and have designed a symbolic algorithm to strategically explore and infer the structure of "finite state" environments. The heart of this algorithm is a clever representation of the environment called an update graph. We have developed a connectionist implementation of the update graph using a highly-specialized network architecture. With back propagation learning and a trivial exploration strategy-choosing random actions-the network can outperform the Rivest and Schapire algorithm on simple problems. Perhaps the most interesting consequence of the connectionist approach is that, by relaxing the constraints imposed by a symbolic description, it suggests a more general representation of the update graph, thus allowing for greater flexibility in expressing potential solutions.
\end{abstract}

Keywords. Finite-state automata, automata induction, update graph, diversity-based inference, connectionism/neural networks, back propagation

Consider a robot placed in an unfamiliar environment. The robot is allowed to explore the environment by performing actions and sensing the resulting environmental state. The robot's task is to construct an internal model of the environment, a model that will allow it to predict the consequences of its actions and to determine what sequences of actions to take to reach particular goal states. This scenario is extremely general; it applies not only to physical environments, but also to abstract and artificial environments such as electronic devices (e.g., a VCR), computer programs (e.g., a text editor), and classical AI problem-solving domains (e.g., blocks world). Any agent-human or computer-that aims to manipulate its environment toward some desired end requires an internal representation of the environment. This is because, in any reasonably complex situation, the agent can directly perceive only a small fraction of the global environmental state at any time; the rest must be stored internally if the agent is to act effectively.

In this paper, we describe a connectionist network that learns the structure of its environment. The network architecture is based on a representation of finite-state automata developed by Rivest and Schapire (1987a, 1987b; Schapire, 1988). We begin by first describing several environments. 


\section{Sample environments}

In each environment, the robot has a set of discrete actions it can execute to move from one environmental state to another. At each environmental state, a set of binary-valued sensations can be detected by the robot. Descriptions of five sample environments follow, the first three of which come from Rivest and Schapire.

\subsection{The n-room world}

The $n$-room world consists of $n$ rooms arranged in a circular chain (Figure 1). Each room is connected to the two adjacent rooms. In each room is a light bulb and a light switch. The robot can sense whether the light in the room where it currently stands is on or off. The robot has three possible actions: move to the next room down the chain, move to the next room up the chain, and toggle the light switch in the current room.

\subsection{The little prince world}

The robot resides on the surface of a $2 \mathrm{D}$ planet. There are four distinct locations on the planet: north, south, east, and west. To the west, there is a rose; to the east, a volcano. The robot has two sensations, one indicating the presence of a rose at the current location, the other a volcano. The robot has available three actions: move to the next location in the direction it is currently facing, move to the next location away from the direction it is facing, and turn its head around to face in the opposite direction.

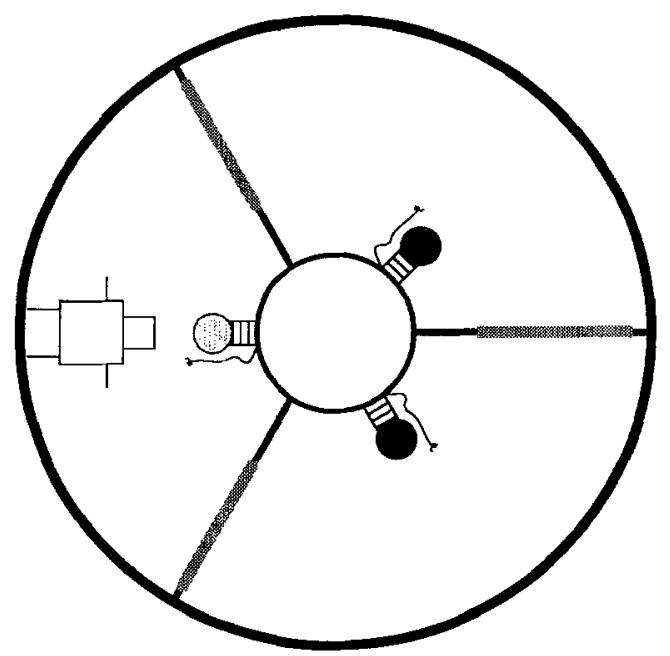

Figure 1. A three-room world. 


\subsection{The car radio world}

The robot manipulates a car radio that can receive three stations, each of which plays a different type of music: top 40, classical, and jazz. The radio has two "preset" buttons labeled X and Y, as well as "forward seek" and "backward seek" buttons. There are three sensations, indicating the type of music played by the current station. The robot has six actions available: recall the station in preset $\mathrm{X}$ or $\mathrm{Y}$, store the current station in preset $\mathrm{X}$ or $Y$, and search forward or backward to the next station from the current station.

\subsection{The grid world}

The grid world consists of an $n \times n$ grid of cells. Half of the cells possess distinct markings. The robot stands in one cell and can sense the marking in that cell, if any. There are thus $n^{2} / 2$ sensations. The robot can take four actions: move to the next cell to the left, to the right, up, or down. Movement off one edge of the grid wraps around to the other side.

\subsection{You figure it out}

The above environments appear fairly simple partially because we have a wealth of world knowledge about light switches, radios, etc. For instance, we know that toggling a light switch undoes the effect of a previous toggle. The robot operates without the benefit of this background knowledge. To illustrate the abstract task that the robot faces, consider an environment with two actions, $A$ and $B$, and one binary-valued sensation. Try to predict the sensations that will be obtained given the following sequence of actions.

Action: $\quad A$ A B B A B A B B B B A B A A A B A B B A A B A B B

Resulting Sensation: $\begin{array}{lllllllllllllllllllllllllll}1 & 0 & 0 & 0 & 1 & 0 & 1 & 1 & 1 & 1 & 1 & 0 & 1 & 0 & 1 & 0 & 0 & 1 & 0 & 1 & ? & ? & ? & ?\end{array}$

If you give up, this is a simplified version of the $n$-room problem with only two rooms. Action A toggles the light switch, B moves from one room to the other, and the sensation indicates the state of the light in the current room. Initially, both lights are assumed to be off. People generally find this problem extremely challenging, if not insurmountably abstract, even when allowed to select actions and observe the consequences.

\section{Modeling the environment with a finite-state automaton}

The environments we wish to consider can be modeled by a finite-state automaton (FSA). Nodes of the FSA correspond to states of an environment. Labeled links connect each node of the automaton to other nodes and correspond to the actions that the robot can execute to move between environmental states. Associated with each node is a set of binary values: the sensations that can be detected by the robot in the corresponding environmental state. 


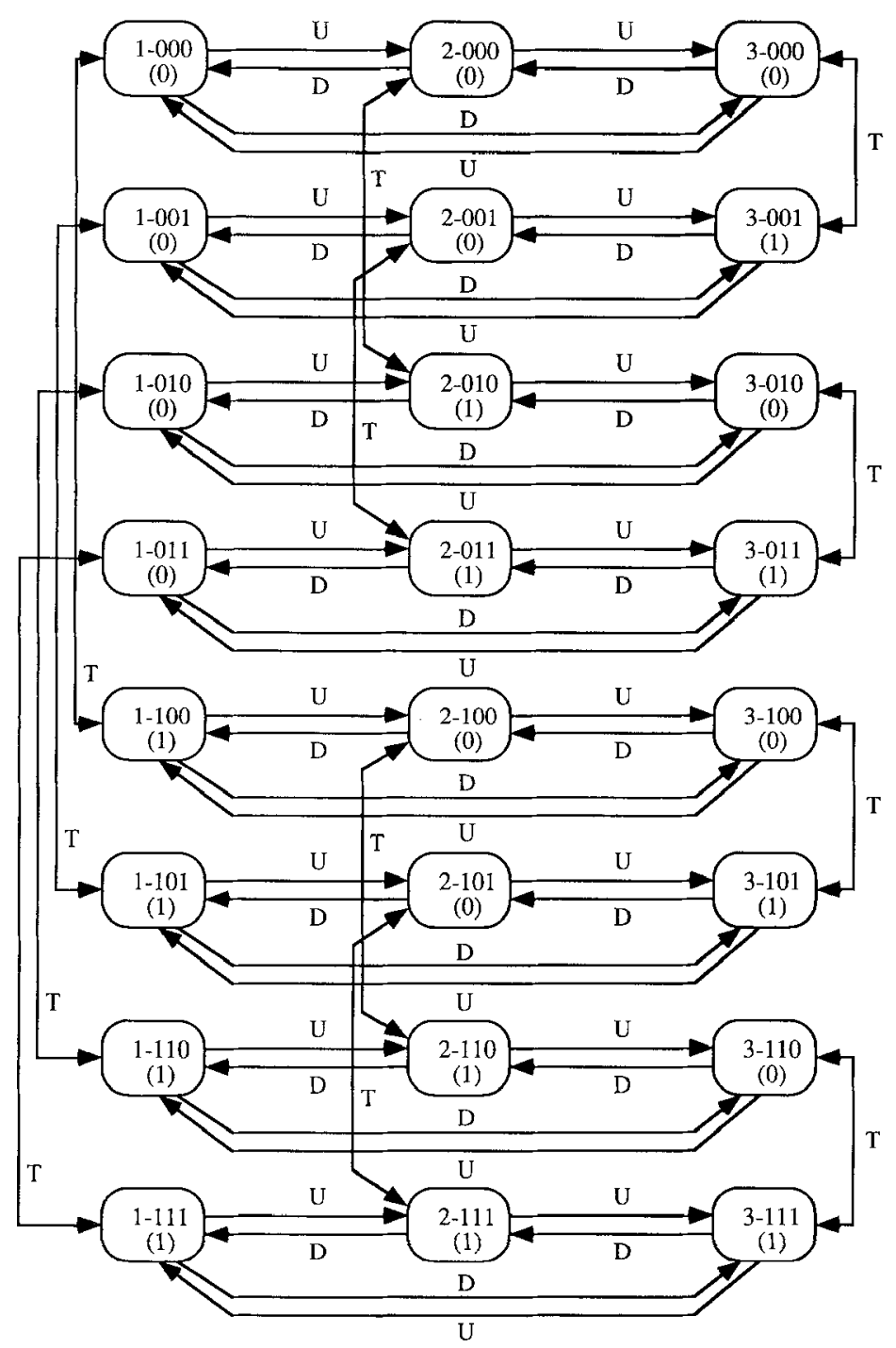

Figure 2. An FSA for the three-room world.

Figure 2 illustrates the FSA for a three-room world. Each node is labeled by the corresponding environmental state, coded in the form $r-s_{1} s_{2} s_{3}$, where $r$ is the current room-1, 2, or 3 -and $s_{i}$ is the status of the light in room $i-0$ for off and 1 for on. The sensation associated with each node is written in parentheses. Links between nodes are labeled with one of the three actions: toggle (T), move up (U), or move down (D).

The FSA represents the underlying structure of the environment. If the FSA is known, one can predict the sensory consequences of any sequence of actions. Further, the FSA can be used to determine a sequence of actions required to obtain a certain goal state. For 
example, if the robot wishes to avoid light, it should follow a link or sequence of links for which the resulting sensation is 0 .

Although one might try developing an algorithm to learn the FSA directly, there are several arguments against doing so (Schapire, 1988): (1) because many FSAs yield the same input/ output behavior, there is no unique FSA; (2) knowing the structure of the FSA is unnecessary because we are only concerned with its input/output behavior; and, most importantly, (3) the FSA often does not capture redundancy inherent in the environment. As an example of this final point, in the $n$-room world, the T action has the same behavior independent of the current room number and the state of the lights in the other rooms, yet in the FSA of Figure 2, knowledge about "toggle" must be encoded for each room and in the context of the particular states of the other rooms. Thus, the simple semantics of an action like $T$ are encoded repeatedly for each of the $n 2^{n}$ distinct states.

\section{Modeling the environment with an update graph}

Rather than trying to learn the FSA, Rivest and Schapire suggest learning another representation of the environment called an update graph. The advantage of the update graph representation is that in environments with many regularities, the number of nodes in the update graph can be much smaller than in the FSA (e.g., $2 n$ versus $n 2^{n}$ for the $n$-room world). ${ }^{1}$

The Appendix summarizes Rivest and Schapire's formal definition of the update graph. Their definition is based on the notion of tests that can be performed on the environment, and the equivalence of different tests. In this section, we present an alternative, more intuitive view of the update graph that facilitates a connectionist interpretation of the graph.

Consider again the three-room world. To model this environment, the essential knowledge required is the status of the lights in the current room (CUR), the next room up from the current room (UP), and the next room down from the current room (DOWN). Assume the update graph has a node for each of these environmental variables. Further assume that each node has an associated value indicating whether the light in the particular room is on or off.

If we know the value of the variables in the current environmental state, what will their new values be after taking some action, say U? The new value of cur becomes the previous value of UP; the new value of DOWN becomes the previous value of CUR; and in the threeroom world, the new value of UP becomes the previous value of Down. As depicted in Figure $3 a$, this action thus results in shifting values around in the three nodes. This makes sense because moving up does not affect the status of any light, but it does alter the robot's position with respect to the three rooms. Figure $3 \mathrm{~b}$ shows the analogous flow of information for the action D. Finally the action T should cause the status of the current room's light to be complemented while the other two rooms remain unaffected (Figure 3c). In Figure 3d, the three sets of links from Figure 3a-c have been superimposed and have been labeled with the associated action.

One final detail: The Rivest and Schapire update graph formalism does not make use of the "complementation" link. To avoid it, one may split each node into two values, one representing the status of a room and the other its complement (Figure 3e). Toggling thus involves exchanging the values of CUR and $\overline{C U R}$. Just as the values of CUR, UP, and DOWN must be shifted for the actions $U$ and $D$, so must their complements. 
(a)

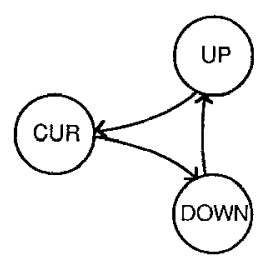

(c)
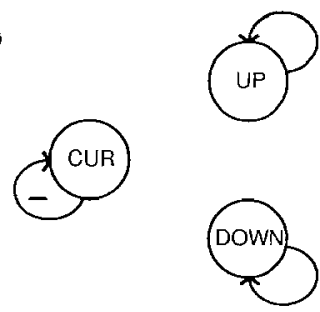

(b)
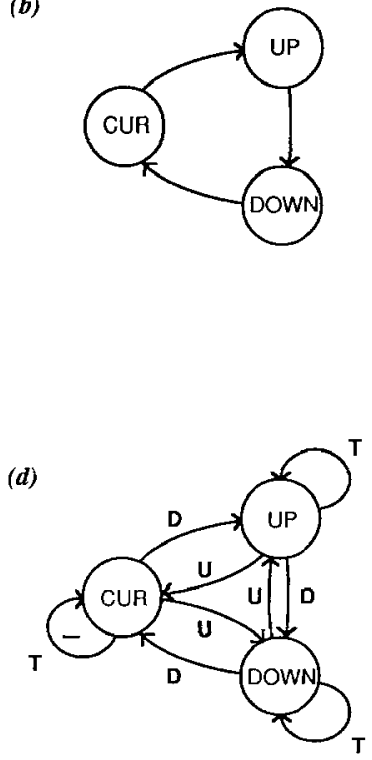

(e)

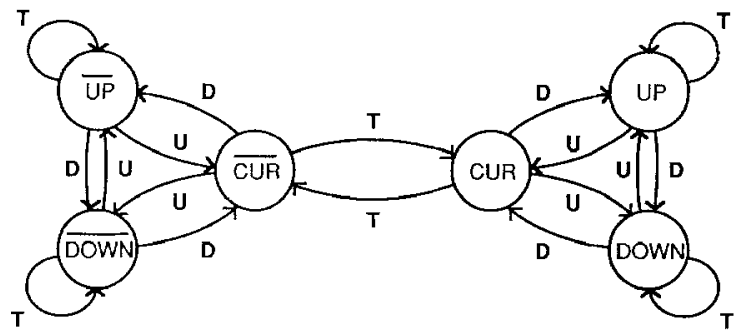

Figure 3. (a) Links between nodes indicating the desired information flow on performing the action U. cUR represents the status of the lights in the current room, up the status of the lights in the next room up, and DowN the status of the lights in the next room down. (b) Links between nodes indicating the desired information flow on performing the action D. (c) Links between nodes indicating the desired information on performing the action $T$. The " -" on the link from cuR to itself indicates that the value must be complemented. (d) Links from the three separate actions superimposed and labeled by the action. (e) The complementation link can be avoided by adding a set of nodes that represent the complements of the original set. This is the update graph for a threeroom world.

Given the update graph in Figure 3e and the value of each node for the current environmental state, the result of any sequence of actions can be predicted simply by shifting values around in the graph. Thus, as far as predicting the input/output behavior of the environment is concerned, the update graph serves the same purpose as the FSA.

For every FSA, there exists a corresponding update graph. In fact, the update graph in Figure 3e might even be viewed as a distributed representation of the FSA in Figure 2. 
In the FSA, each environmental state is represented by one "active" node. In the update graph, each environmental state is represented by a pattern of activity across the nodes.

One defining and nonobvious (from the current description) property of an update graph is that each node has exactly one incoming link for each action. For example, cur gets input from $\overline{\mathrm{CUR}}$ for the action $\mathrm{T}$, from UP for $\mathrm{U}$, and from DOWN for $\mathrm{D}$. Table 1 , which represents the update graph in a slightly different manner, provides another way of describing the unique-input property. The table shows node connectivity in the update graph, with a " 1 " indicating that two nodes are connected for a particular action, and " 0 " for no connection. The unique-input property specifies that there must be exactly one non-zero value in each row of each matrix.

Table 1. Alternative representation of update graph.

\begin{tabular}{|c|c|c|c|c|c|c|}
\hline \multicolumn{7}{|c|}{ Update Graph Connectivity for Move Up } \\
\hline \multirow[b]{2}{*}{ To Node } & \multicolumn{6}{|c|}{ From Node } \\
\hline & CUR & UP & DowN & $\overline{\text { CUR }}$ & $\overline{\mathrm{UP}}$ & $\overline{\text { DOWN }}$ \\
\hline CUR & 0 & 1 & 0 & 0 & 0 & 0 \\
\hline UP & 0 & 0 & 1 & 0 & 0 & 0 \\
\hline DOWN & 1 & 0 & 0 & 0 & 0 & 0 \\
\hline$\overline{\text { CUR }}$ & 0 & 0 & 0 & 0 & 1 & 0 \\
\hline$\overline{\mathrm{UP}}$ & 0 & 0 & 0 & 0 & 0 & 1 \\
\hline$\overline{\text { DOWN }}$ & 0 & 0 & 0 & 1 & 0 & 0 \\
\hline \multicolumn{7}{|c|}{ Update Graph Connectivity for Move Down } \\
\hline \multirow[b]{2}{*}{ To Node } & \multicolumn{6}{|c|}{ From Node } \\
\hline & CUR & UP & DOWN & $\overline{\mathrm{CUR}}$ & $\overline{\mathbf{U P}}$ & $\overline{\text { DOWN }}$ \\
\hline CUR & 0 & 0 & 1 & 0 & 0 & 0 \\
\hline UP & 1 & 0 & 0 & 0 & 0 & 0 \\
\hline DOWN & 0 & 1 & 0 & 0 & 0 & 0 \\
\hline$\overline{\text { CUR }}$ & 0 & 0 & 0 & 0 & 0 & 1 \\
\hline$\overline{\mathrm{UP}}$ & 0 & 0 & 0 & 1 & 0 & 0 \\
\hline$\overline{\text { DOWN }}$ & 0 & 0 & 0 & 0 & 1 & 0 \\
\hline
\end{tabular}

\begin{tabular}{lcccccc}
\hline \multicolumn{7}{c}{ Update Graph Connectivity for Toggle } \\
\hline \multirow{7}{*}{ To Node } & CUR & UP & DOWN & $\overline{\text { CUR }}$ & $\overline{\text { UP }}$ & $\overline{\text { DOWN }}$ \\
\cline { 2 - 7 } CUR & 0 & 0 & 0 & 1 & 0 & 0 \\
UP & 0 & 1 & 0 & 0 & 0 & 0 \\
DOWN & 0 & 0 & 1 & 0 & 0 & 0 \\
$\overline{\text { CUR }}$ & 1 & 0 & 0 & 0 & 0 & 0 \\
UP & 0 & 0 & 0 & 0 & 1 & 0 \\
DOWN & 0 & 0 & 0 & 0 & 0 & 1 \\
\hline
\end{tabular}




\subsection{The Rivest and Schapire algorithm}

Rivest and Schapire have developed a symbolic algorithm (hereafter, the RS algorithm) to strategically explore an environment and learn its update graph representation. They break the learning problem into two steps: (a) inferring the structure of the update graph, and (b) maneuvering the robot into an environmental state where the value of each node is known. Step (b) is relatively straightforward. Step (a) involves a method of experimentation to determine whether pairs of action sequences are equivalent in terms of their sensory outcomes. For a special class of environments, permutation environments, in which each action sequence has an inverse, the RS algorithm can infer the environmental structurewithin an acceptable margin of error-by performing a number of actions polynomial in the number of update graph nodes and the number of alternative actions.

This polynomial bound is impressive, but in practice, Schapire (personal communication) achieves reasonable performance only by including heuristics that attempt to make better use of the information provided by the environment. To elaborate, the RS algorithm formulates explicit hypotheses about regularities in the environment and tests these hypotheses one or a relatively small number at a time. As a result, the algorithm may not make full use of the environmental feedback obtained. It thus seems worthwhile to consider alternative approaches that allow more efficient use of the environmental feedback, and hence, more efficient learning of the update graph. We have pursued a connectionist approach, which has shown quite promising results in preliminary experiments as well as a number of other powerful advantages. We detail these advantages below, but must first describe the basic approach.

\section{Viewing the update graph as a connectionist network}

SLUG is a connectionist network that performs subsymbolic learning of update graphs. Before the learning process itself can be described, however, we must first consider the desired outcome of learning. That is, what should SLUG look like following training if it is to behave as an update graph? Start by assuming one unit in SLUG for each node in the update graph. The activity level of the unit represents the boolean value associated with the update graph node. Some of these units serve as "outputs" of SLUG. For example, in the three-room world, the output of SLUG is the unit that represents the status of the current room. In other environments, there may be several sensations (e.g., the little prince world), in which case several output units are required.

What is the analog of the labeled links in the update graph? The labels indicate that values are to be sent down a link when a particular action occurs. In connectionist terms, the links should be gated by the action. To elaborate, we might include a set of units that represent the possible actions; these units act to multiplicatively gate the flow of activity between units in the update graph. Thus, when a particular action is to be performed, the corresponding action unit is activated, and the connections that are gated by this action become enabled.

If the action units form a local representation, i.e., only one is active at a time, exactly one set of connections is enabled at a time. Consequently, the gated connections can be replaced by a set of weight matrices, one per action (like those shown in Table 1). To predict 
the consequences of a particular action, say $T$, the weight matrix for $T$ is simply plugged into the network and activity is allowed to propagate through the connections. Thus, SLUG is dynamically rewired contingent on the current action.

The effect of activity propagation should be that the new activity of a unit is the previous activity of some other unit. A linear activation function is sufficient to achieve this:

$$
\mathbf{x}(t)=\mathbf{W}_{a(t)} \mathbf{x}(t-1),
$$

where $a(t)$ is the action selected at time $t, \mathbf{W}_{a(t)}$ is the weight matrix associated with this action, and $\mathbf{x}(t)$ is the activity vector that results from taking action $a(t)$. Assuming weight matrices like those shown in Table 1 , which have zeroes in each row except for one connection of strength 1 , the activiation rule will cause activity values to be copied around the network. Although linear activation functions are generally not appropriate for back propagation applications (Rumelhart, Hinton, \& Williams, 1986), the architecture here permits such a simple function. SLUG is thus a linear system, which is extremely useful because it allows us to use the tools and methods of linear algebra for analyzing network behavior, as we show below.

\section{Training SLUG}

We have described how SLUG could be hand-wired to behave as an update graph, and now turn to the procedure used to learn the appropriate connection strengths. For expository purposes, assume that the number of units in the update graph is known in advance (this is not necessary, as we show below). A weight matrix is required for each action, with a potential non-zero connection between every pair of units. As in most connectionist learning procedures, the weight matrices are initialized to random values; the outcome of learning will be a set of matrices like those in Table 1 .

If the network is to behave as an update graph, the critical constraint on the connectivity matrices is that each row of each weight matrix should have connection strengths of zero except for one value which is 1 (assuming Equation 1). To achieve this property, additional constraints are placed on the weights. We have explored a combination of three constraints:

(1) $\sum_{j} w_{a i j}^{2}=1$,

(2) $\sum_{j} w_{a i j}=1$, and

(3) $w_{a i j} \geq 0$,

where $w_{a i j}$ is the connection strength to $i$ from $j$ for action $a$. If all three of these constraints are satisfied, the incoming weights to a unit are guaranteed to be all zeros except for one value which is 1 . This can be intuited from Figure 4, which shows the constraints in a two-dimensional weight space. Constraint 1 requires that vectors lie on the unit circle, 


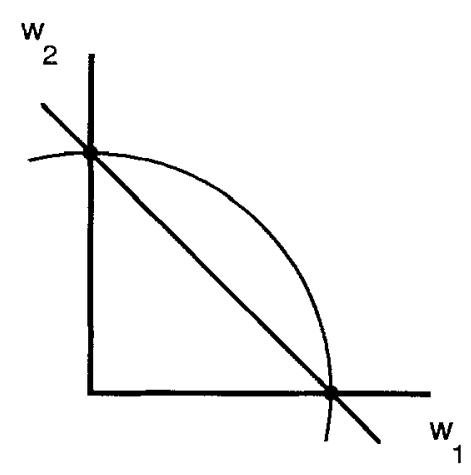

Figure 4. A two-dimensional space representing the weights, $w_{1}$ and $w_{2}$, feeding into a 2-input unit. The circle and line indicate the subregions specified by constraints 1 and 2 , respectively. The two points of intersection are $(1,0)$ and $(0,1)$.

constraint 2 requires that vectors lie along the line $w_{1}+w_{2}=1$, and constraint 3 requires that vectors lie in the first quadrant. Constraint 3 is redundant in a two-dimensional weight space but becomes necessary in higher dimensions.

Constraint 1 is satisfied by introducing a secondary error term,

$$
E_{s e c}=\sum_{a, i}\left(1-\left\|\mathbf{w}_{a i}\right\|\right)^{2}
$$

where $\mathbf{w}_{a i}$ is the incoming weight vector to unit $i$ for action $a$. The learning procedure attempts to minimize this error along with the primary error asociated with predicting environmental sensations. Constraints 2 and 3 are rigidly enforced by renormalizing the $\mathbf{w}_{a i}$ following each weight update. The normalization procedure finds the shortest distance projection from the updated weight vector to the hyperplane specified by constraint 2 that also satisfies constraint 3 .

\subsection{Details of the training procedure}

Initially, a random weight matrix is generated for each action. Weights are selected from a uniform distribution in the range $[0,1]$ and are normalized to satisfy constraint 2 . At each time step $t$, the following sequence of events transpires:

1. An action, $a(t)$, is selected at random.

2. The weight matrix for that action, $\mathbf{W}_{a(t)}$, is used to compute the activities at $t, \mathbf{x}(t)$, from the previous activities $\mathbf{x}(t-1)$.

3. The selected action is performed on the environment and the resulting sensations are observed.

4. The observed sensations are compared with the sensations predicted by SLUG (i.e., the activities of units chosen to represent the sensations) to compute a measure of error. To this error is added the contribution of constraint 1 . 
5. The back propagation "unfolding-in-time" procedure (Rumelhart, Hinton, \& Williams, 1986) is used to compute the derivative of the error with respect to weights at the current and earlier time steps, $\mathbf{W}_{a(t-i)}$, for $i=0 \ldots \tau-1$.

6. The weight matrices for each action are updated using the overall error gradient and then are renormalized to enforce constraints 2 and 3 .

7. The temporal record of unit activities, $\mathbf{x}(t-i)$ for $i=0 \ldots \tau$, which is maintained to permit back propagation in time, is updated to reflect the new weights. (See further explanation below.)

8. The activities of the output units at time $t$, which represent the predicted sensations, are replaced by the observed sensations. ${ }^{2}$

Steps 5-7 require further elaboration. The error measured at step $t$ may be due to incorrect propagation of activities from step $t-1$, which would call for modification of the weight matrix $\mathbf{W}_{a(t)}$. But the error may also be attributed to incorrect propagation of activities at earlier times. Thus, back propagation is used to assign blame to the weights at earlier times. One critical parameter of training is the amount of temporal history, $\tau$, to consider. We have found that, for a particular problem, error propagation beyond a certain critical number of steps does not improve learning performance, although any fewer does indeed harm performance. In the results described below, we generally set $\tau$ for a particular problem to what appeared to be a safe limit: one less than the number of nodes in the update graph solution of the problem.

To back propagate error in time, a temporal record of unit activities is maintained. However, a problem arises with these activities following a weight update: the activities are no longer consistent with the weights-i.e., Equation 1 is violated. Because the error derivatives computed by back propagation are exact only when Equation 1 is satisfied, future weight updates based on the inconsistent activities are not assured of being correct. Empirically, we have found the algorithm extremely unstable if we do not address this problem.

In most situations where back propagation is applied to temporally-extended sequences, the sequences are of finite length. Consequently, it is possible to wait until the end of the sequence to update the weights, at which point consistency between activities and weights no longer matters because the system starts fresh at the beginning of the next sequence. In the present situation, however, the sequence of actions does not terminate. We thus were forced to consider alternative means of ensuring consistency. One approach we tried involved updating the weights only after every, say, 25 steps. Immediately following the update, the weights and activities are inconsistent, but after $\tau$ steps (when the inconsistent activities drop off the activity history record), consistency is once again achieved. A more successful approach involved updating the activities after each weight change to force consistency (step 7 of the list above). To do this, we propagated the earliest activities in the temporal record, $\mathbf{x}(t-\tau)$, forward again to time $t$, using the updated weight matrices. ${ }^{3}$

The issue of consistency arises because at no point in time is SLUG instructed as to the state of the environment. That is, instead of being given an activity vector as input, part of SLUG's learning task is to discover the appropriate activity vector. This might suggest a strategy of explicitly learning the activity vector, that is, performing gradient descent in both the weight space and activity space. However, our experiments indicate that this strategy does not improve SLUG's performance. One plausible explanation is the following. 
If we perform gradient descent in weight space based on the error from a single trial, and then force activity-weight consistency, the updated output unit activities are guaranteed to be closer to the target values (assuming a sufficiently small learning rate and that the weight constraints have minor influence). Thus, the effect of this procedure is to reduce the error in the observable components of the activity vector, which is similar to performing gradient descent in activity space directly.

A final comment regarding the training procedure: In our simulations, learning performance was better with target activity levels of -1 and +1 (for light is off and on, respectively) rather than 0 and 1 . One explanation for this is that random activations and random (nonnegative) connection strengths tend to cancel out in the $-1 /+1$ case, but not in the $0 / 1$ case.

\section{Results}

SLUG's architecture, dynamics, and training procedure are certainly nonstandard and nonintuitive. Our original experiments in this domain involved more standard recurrent connectionist architectures (e.g., Elman, 1988; Mozer, 1989) and were spectacularly unsuccessful. Many simple environments could not be learned, predictions were often inaccurate, and a great deal of training was required. Rivest and Schapire's update graph representation has thus proven beneficial in the development of SLUG. It provides strong constraints on network architecture, dynamics, and training procedure.

Figure 5 shows the weights in SLUG for the three-room world at three stages of learning. The "step" refers to how many actions the robot has taken, or equivalently, how many times the weights have been updated. The bottom diagram in the figure depicts a connectivity pattern identical to that presented in Table 1, and corresponds to the update graph of Figure 3e. To explain the correspondence, think of the diagram as being in the shape of a person who has a head, left and right arms, left and right legs, and a heart. For the action $U$, the head-the output unit-receives input from the left leg, the left leg from the heart, and the heart from the head, thereby forming a three-unit loop. The other three unitsthe left arm, right arm, and right leg-form a similar loop. For the action $D$, the same two loops are present but in the reverse direction. These two loops also appear in Figure 3e. For the action $T$, the left and right arms, heart, and left leg each keep their current value, while the head and the right leg exchange values. This corresponds to the exchange of values between the CUR and $\overline{\text { CUR }}$ nodes of the Figure $3 \mathrm{e}$.

In addition to learning the update graph connectivity, SLUG has simultaneously learned the correct activity values associated with each node for the current state of the environment. Armed with this information, SLUG can predict the outcome of any sequence of actions. Indeed, the prediction error drops to zero, causing learning to cease and SLUG to become completely stable. Because the ultimate weights and activities are boolean, SLUG can predict infinitely far into the future with no degradation in performance (cf., ServanSchreiber, Cleeremans, \& McClelland, 1988).

Now for the bad news: SLUG does not converge for every set of random initial weights, and when it does, it requires on the order of 6,000 steps, much greater than the RS algorithm. ${ }^{4}$ However, when the weight constraints are removed, SLUG converges without fail and in about 300 steps. It appears that only in extremely small environments do the weight 
Step 0

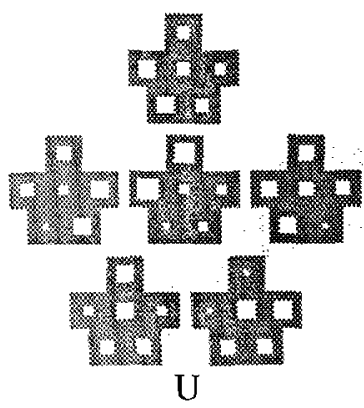

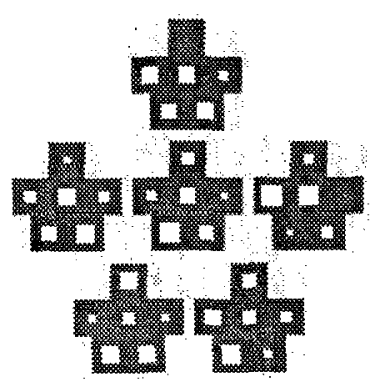

D

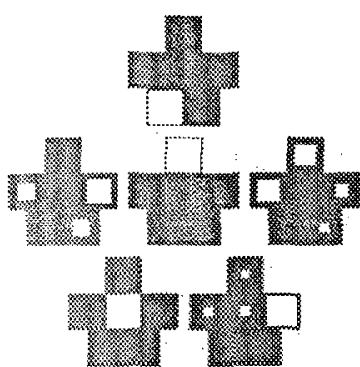

U

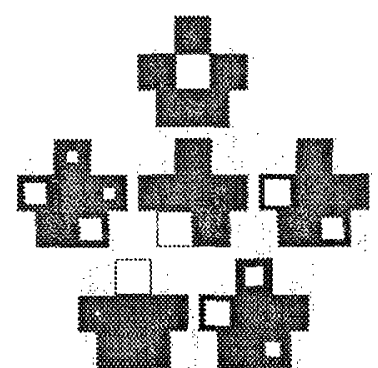

D

Step 6000

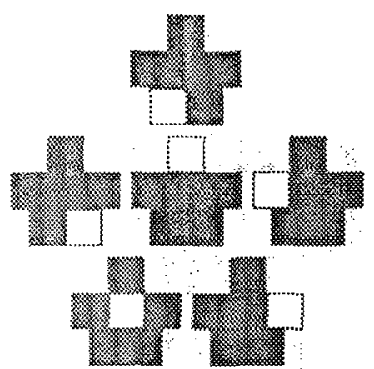

U

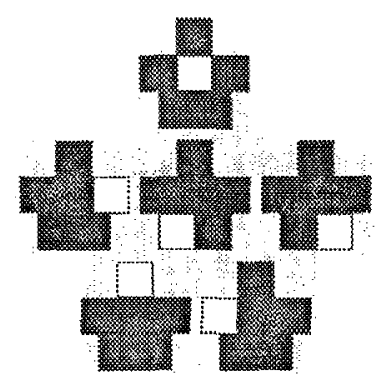

D

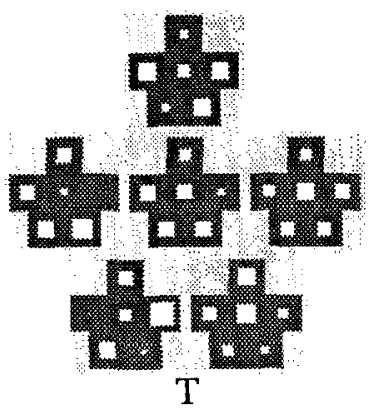

$\mathrm{T}$
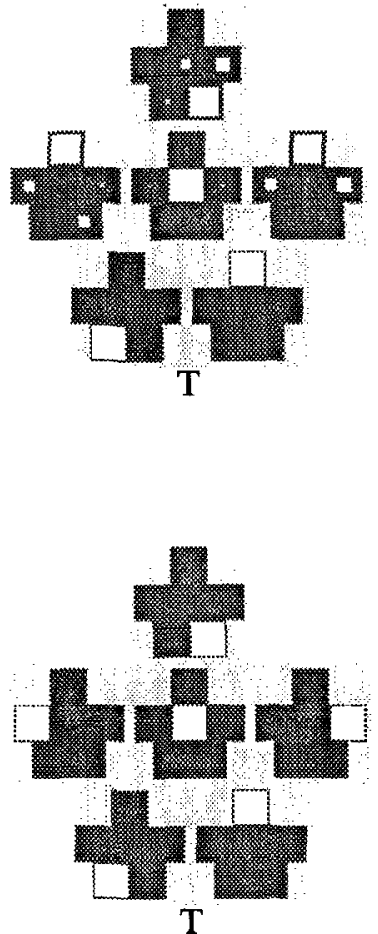

Figure 5. The three-room world. Weights learned by SLUG with six units at three stages of learning: step 0 reflects the initial random weights, step 3000 reflects the weights midway through learning, and step 6000 reflects the weights upon completion of learning. Each large diagram represents the weights corresponding to one of the three actions. Each small diagram contained within a large diagram represents the connection strengths feeding into a particular unit for a particular action. There are six units, hence six small diagrams. The output unit, which indicates the state of the light in the current room, is the protruding "head" of the large diagram. A white square in a particular position of a small diagram represents the strength of connection from the unit in the homologous position in the large diagram to the unit represented by the small diagram. The area of the square is proportional to the connection strength. 
constraints help SLUG discover a solution. We consider below why the weight constraints are harmful and possible remedies.

Without weight constraints, there are two problems. First, the system has difficulty converging onto an exact solution. One purpose that the weight constraints serve is to lock in on a set of weights when the system comes sufficiently close; without the constraints, we found it necessary to scale the learning rate in proportion to the mean-squared prediction error to avoid overstepping solutions. Second the resulting weight matrix, which contains a collection of positive and negative weights of varying magnitudes, is not readily interpreted (see Figure 6). In the case of the three-room world, one reason why the final weights are difficult to interpret is because the net has discovered a solution that does not satisfy the update graph formalism; it has discovered the notion of complementation links of the sort shown in Figure 3d. With the use of complementation links, only three units are required, not six. Consequently, the three unnecessary units are either cut out of the solution or encode information redundantly. SLUG's solutions are much easier to understand when the network consists of only three units. Figure 7 depicts one such solution, which corresponds to the graph in Figure 3d. SLUG also discovers other solutions in which two of the three connections in the three-unit loop are negative, one negation cancelling out the effect of the other. Allowing complementation links can halve the number of update
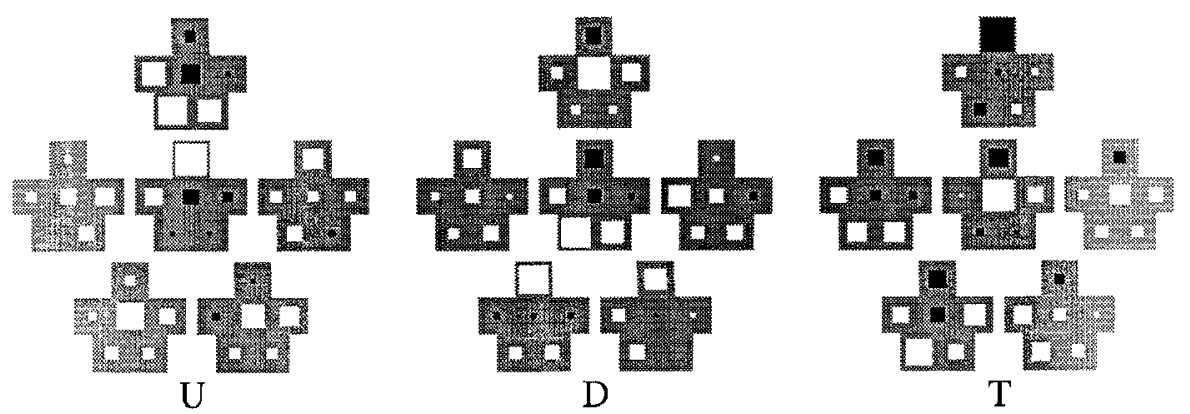

Figure 6 The three-room world. Weights learned by SLUG with six units without weight constraints. Black squares indicate negative weights, white positive.
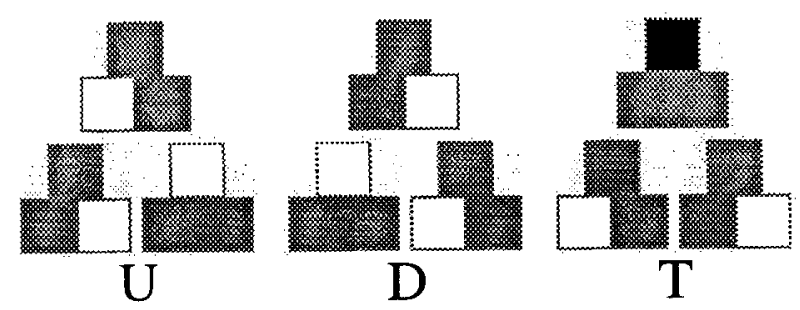

Figure 7. The three-room world. Weights learned by SLUG with three units without weight constraints. (The weights have been cleaned up slightly to make the result clearer.) 
Table 2. Number of steps required to learn update graph.

\begin{tabular}{lcr}
\hline \multicolumn{1}{c}{ Environment } & RS Algorithm & SLUG \\
\hline Little Prince World & 200 & 91 \\
Three-Room World & not available & 298 \\
Four-Room World & 1,388 & 1,509 \\
6×6 Grid World & not available & 8,142 \\
Car Radio World & 27,695 & 8,167 \\
32-Room World & 52,436 & fails \\
\hline
\end{tabular}

graph nodes required for many environments. This is one fairly direct extension of Rivest and Schapire's update graph formalism that SLUG suggests.

Table 2 compares the performance of the RS algorithm against that of SLUG without weight constraints for a sampling of environments. ${ }^{5}$ Performance is measured in terms of the number of actions the robot must take before it is able to predict the outcome of subsequent actions, that is, the number of actions required to learn the update graph structure and the truth value associated with each node. The performance data reported for SLUG was the median over 25 replications of each simulation. SLUG was considered to have learned the task on a given trial if the correct predictions were made for at least the next 2,500 steps.

The learning rates used in our simulations were adjusted dynamically every 100 steps by averaging the current learning rate with a rate proportional to the mean squared error obtained on the last 100 steps. Several runs were made to determine what initial learning rate and constant of proportionality yielded the best performance. It turned out that performance was relatively invariant under a wide range of these parameters. Momentum did not appear to help. 6

In simple environments, the connectionist update graph can outperform the RS algorithm. These results are quite surprising when considering that the action sequence used to train SLUG is generated at random, in contrast to the RS algorithm, which involves a strategy for exploring the environment. We conjecture that SLUG does as well as it does because it considers and updates many hypotheses in parallel at each time step. That is, after the outcome of a single action is observed, nearly all weights in SLUG are adjusted simultaneously.

In complex environments-ones in which the number of nodes in the update graph is quite large and the number of distinguishing environmental sensations is relatively smallSLUG does poorly. As an example of such, a 32-room world cannot be learned by SLUG whereas the RS algorithm succeeds. An intelligent exploration strategy seems necessary in complex environments: with a random exploration strategy, the time required to move from one state to a distant state becomes so large that links between the states cannot be established.

The 32-room world is extreme; all rooms are identical and the available sensory information is meager. Such an environment is quite unlike natural environments, which provide a relative abundance of sensory information to distinguish among environmental states. SLUG performs much better when more information about the environment can be sensed directly. For example, learning the 32-room world is trivial if SLUG is able to sense the 
states of all 32 rooms at once (the median number of steps to learn is only 1,209). The $6 \times 6$ grid world is another environment as large as the 32-room world in terms of the number of nodes SLUG requires, but it is much easier to learn because of the rich sensory information.

\subsection{Noisy environments}

The RS algorithm is not designed to handle environments with stochastic sensations. In contrast, SLUG's performance degrades gracefully in the presence of noise. For example, SLUG is able to learn the update graph for the little-prince world even when sensations are unreliable, say, when sensations are registered incorrectly $10 \%$ of the time. To train SLUG properly in noisy environments, however, the training procedure must be altered. If the observed sensation replaces SLUG's predicted sensation and the observed sensation is incorrectly registered, the values of nodes in the graph are disrupted and SLUG requires several noise-free steps to recover. Thus, a procedure might be used in which the predicted sensation is not completely replaced by the observed sensation, but rather some average of the two is computed; additionally, the average should be weighted towards the prediction as SLUG's performance improves.

\subsection{Prior specification of update graph size}

The RS algorithm requires an upper bound on the number of nodes in the update graph. The results presented in Table 2 are obtained when the RS algorithm knows exactly how many nodes are required in advance. The algorithm fails if it is given an upper bound less than the required number of nodes, and performance degrades as the upper bound increases above the required number. SLUG will also fail if it is given fewer units than are necessary for the task. However, performance does not appear to degrade as the number of units increases beyond the minimal number. Table 3 presents the median number of steps required

Table 3. Median number of steps to learn four-room world.

\begin{tabular}{cc}
\hline Units in SLUG & $\begin{array}{c}\text { Number of Steps to } \\
\text { Learn Update Graph }\end{array}$ \\
\hline 4 & 2,028 \\
6 & 1,380 \\
8 & 1,509 \\
10 & 1,496 \\
12 & 1,484 \\
14 & 1,630 \\
16 & 1,522 \\
18 & 1,515 \\
20 & 1,565 \\
\hline
\end{tabular}


to learn the four-room world as the number of units in SLUG is varied. Although performance is independent of the number of units here, extraneous units greatly improve performance when the weight constraints are applied. Only 3 of 25 replications of the four-room world simulation with 8 units and weight constraints successfully learned the update graph (the simulation was terminated after 100,000 steps), whereas 21 of 25 replications succeeded when 16 units were used.

\subsection{Generalizing the update graph formalism}

Having described the overall performance of SLUG, we return to the issue of why weight constraints appear to harm performance. One straightforward explanation is that there are many possible solutions, only a small number of which correspond to update graphs. With weight constraints, SLUG is prevented from finding alternative solutions. One example of an alternative solution is the network with complementation links presented in Figure 7. Allowing complementation links can halve the number of update graph nodes required for many environments.

An even more radical generalization of the update graph formalism arises from the fact that SLUG is a linear system. Consider a set of weight matrices, $\left\{\mathbf{W}_{a}\right\}$, that correspond to a particular update graph, i.e., each row of each matrix satisfies the constraint that all entries are zero except for one entry that is one (as in Table 1). Further, assume the vector $\mathbf{x}$ indicates the current values of each node. Given the previously-stated activation rule,

$$
\mathbf{x}(t)=\mathbf{W}_{a(t)} \mathbf{x}(t-1)
$$

one can construct an equivalent system,

$$
\mathbf{x}^{\prime}(t)=\mathbf{W}_{a(t)}^{\prime} \mathbf{x}^{\prime}(t-1)
$$

by substituting

$$
\mathbf{x}^{\prime}(t) \equiv \mathbf{Q x}(t)
$$

and

$$
\mathbf{W}_{a(t)}^{\prime} \equiv \mathbf{Q} \mathbf{W}_{a(t)} \mathbf{Q}^{*}
$$

Here, the $\mathbf{W}_{a}$ have dimensions $n \times n$, the $\mathbf{W}_{a}^{\prime}$ have dimensions $m \times m, \mathbf{Q}$ is any matrix of dimensions $m \times n$ and rank $n$, and $\mathbf{Q}^{*}$ is the left inverse of $\mathbf{Q}$. The transformed system consisting of $\mathbf{x}^{\prime}$ and the $\mathbf{W}_{a}^{\prime}$ is isomorphic to the original system; one can determine a unique $\mathbf{x}$ for each $\mathbf{x}^{\prime}$, and hence one can predict sensations in the same manner as before. However, the transformed system is different in one important respect: The $\mathbf{W}_{a}^{\prime}$ do not satisfy the one-nonzero-weight-per-row constraint.

Because the set of matrices $\mathbf{Q}$ that meet our requirements is infinite, so is the number of $\left\{\mathbf{W}_{a}^{\prime}\right\}$ for each $\left\{\mathbf{W}_{a}\right\}$. A network being trained without weight constraints is free to 
discover virtually any of these $\left\{\mathbf{W}_{a}^{\prime}\right\}$ (the only restriction being that the mapping from $\mathbf{x}^{\prime}$ to $\mathbf{x}$ is the identity for the output units, because error is computed from $\mathbf{x}^{\prime}$ directly, not $\mathbf{x})$. Thus, each solution $\left\{\mathbf{W}_{a}\right\}$ that meets the formal definition of an update graph is only a special case-with $\mathbf{Q}$ being the identity matrix - of the more general $\left\{\mathbf{W}_{a}^{\prime}\right\}$. Requiring SLUG to discover this particular solution can complicate learning, as we observed when training SLUG with weight constraints.

If the connectivity restrictions on $\mathbf{W}$ that define an update graph could be generalized to $\mathbf{W}^{\prime}$, these generalized restrictions could be applied to discover a large set of solutions that nonetheless correspond to an update graph. Unfortunately, it does not appear that the restrictions can be mapped in any straightforward way.

An alternative approach we have considered is to train SLUG to discover a solution $\left\{\mathbf{W}_{a}^{\prime}\right\}$ which can then be decomposed into an update graph $\left\{\mathbf{W}_{a}\right\}$ and a transformation matrix $\mathbf{Q}$. The decomposition could be attempted post hoc, but our experiments thus far have consisted of explicitly defining $\mathbf{W}^{\prime}$ in terms of $\mathbf{Q}$ and $\mathbf{W}$. That is, an error gradient is computed with respect to $\mathbf{W}^{\prime}$, which is then translated to gradients with respect to $\mathbf{Q}$ and $W$ ? In addition, the previously-described constraints on $W$ are applied. Although SLUG must still discover the update graph $\left\{\mathbf{W}_{a}\right\}$, we hoped that introducing $\mathbf{Q}$ would allow alternative paths to the solution. This method did help somewhat, but unfortunately, performance did not reach the same levels as training with no weight constraints whatsoever. Figure 8 shows one solution obtained by SLUG under this training regimen.
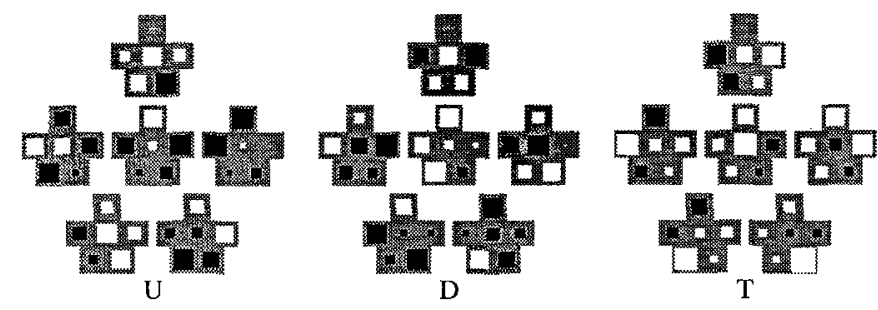

$\mathrm{W}^{\prime}$

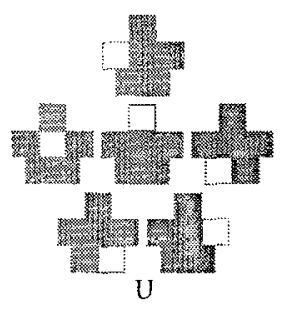

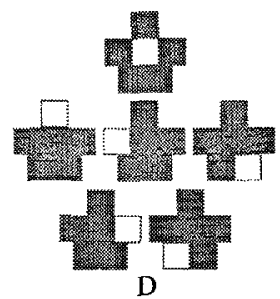

W

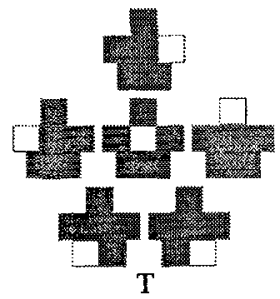

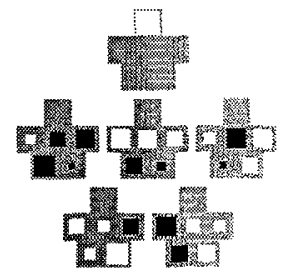

Q

Figure 8. The three-room world. The $\left\{\mathbf{W}_{a}^{\prime}\right\}$ are weight matrices learned by SLUG with six units (along with an activity vector, $\left.\mathbf{x}^{\prime}\right)$. Although the weights do not appear to correspond to an update graph, they in fact can be decomposed into matrices $\mathbf{Q}$ and $\left\{\mathbf{W}_{a}\right\}$ according to $\mathbf{W}_{a}^{\prime}=\mathbf{Q} \mathbf{W}_{a} \mathbf{Q}^{*}$. 


\section{Conclusion}

The connectionist approach to the problem of inferring the structure of a finite-state environment has two fundamental problems that must be overcome if it is to be considered seriously as an alternative to the symbolic approach. First, using a random exploration strategy, SLUG has no hope of scaling to complex environments. An intelligent exploration strategy could potentially be incorporated to force the robot into states where SLUG's predictive abilities are poor. (For a related approach, see Cohn et al., 1990). Second, our greatest successes have occurred when we allowed SLUG to discover solutions that are not necessarily isomorphic to an update graph. One virtue of the update graph formalism is that it is relatively easy to interpret; the same cannot generally be said of the continuous-valued weight matrices discovered by SLUG. However, as we discussed, there is promise of developing methods for transforming the large class of formally equivalent solutions available to SLUG into the more localist update graph formalism to facilitate interpretation.

On a positive note, the connectionist approach has shown several benefits.

- Connectionist learning algorithms provide the potential of parallelism. After the outcome of a single action is observed, nearly all weights in the network are adjusted simultaneously. In contrast, the RS algorithm performs actions in order to test one or a small number of hypotheses, say, whether two particular nodes should be connected. A further example of SLUG's parallelism is that it learns the update graph structure at the same time as the appropriate unit activations, whereas the RS algorithm approaches the two tasks sequentially.

- Performance of the learning algorithm appears insensitive to prior knowledge of the number of nodes in the update graph being learned. As long as SLUG is given at least as many units as required, the presence of additional units does not impede learning. SLUG either disconnects the unnecessary units from the graph or uses multiple units to encode information redundantly. In constast, the RS algorithm requires an upper bound on the update graph complexity, and performance degrades significantly if the upper bound isn't tight.

- During learning, SLUG continually makes predictions about what sensations will result from a particular action. These predictions gradually improve with experience, and even before learning is complete, the predictions can be substantially correct. The RS algorithm cannot make predictions based on its partially constructed update graph. Although the algorithm could perhaps be modified to do so, there would be an associated cost.

- Connectionist learning algorithms are able to accommodate environments in which the sensations are somewhat unreliable. The original RS algorithm was designed for deterministic environments.

- Treating the update graph as matrices of connection strengths has suggested generalizations of the update graph formalism that don't arise from a more traditional analysis. We presented two generalizations. First, there is the fairly direct extension of allowing complementation links. Second, because SLUG is a linear system, any rank-preserving linear transform of the weight matrices will produce an equivalent system, but one that does not have the local connectivity of the update graph. Thus, one can view the Rivest and Schapire update graph formalism as one example of a much larger class of equivalent 
solutions that can be embodied in a connectionist network. While many of these solutions do not obey constraints imposed by a symbolic description (e.g., all-or-none links between nodes), they do yield equivalent behavior. By relaxing the symbolic constraints, the connectionist representation allows for greater flexibility in expressing potential solutions.

We emphatically do not claim that the connectionist approach supercedes the impressive work of Rivest and Schapire. However, it offers complementary strengths and an alternative conceptualization of the learning problem.

\section{Appendix}

In explaining the update graph, we informally characterized the nodes of the graph as representing sensations in the environment relative to the observer's current position. However, the nodes have a formal semantics in Rivest and Schapire's derivation of the update graph, which we present in this Appendix after first introducing a bit more terminology.

A test on the environment can be defined as a sequence of zero or more actions followed by a sensation. A test is performed by executing the sequence of actions from the current environmental state and then detecting the resulting sensation. For example, in the $n$-room world, some tests include: UU? (move up twice and sense the state of the light), UTD? (move up, toggle the light, move down, and then sense the state of the light), ? (sense the state of the light in the current room). The value of a test is the value of the resulting sensation. ${ }^{8}$

Certain tests are equivalent. For example, in the three-room world UU? and D? will always yield the same outcome, independent of the current environmental state. This is because moving up twice will land the robot in the same room as moving down once, so the resulting sensation will be the same. The tests UUUUU?, TUU?, DUTDDDD? are also equivalent to UU? and D?. The set of equivalent tests defines an equivalence class. Rivest and Schapire call the number of equivalence classes the diversity of the environment. The $n$-room world has a diversity of $2 n$, arising from there being $n$ lights that can be sensed, either in their current state or toggled. Each node in the update graph corresponds to one test equivalence class.

The directed, labeled links of the update graph arise from relations among equivalence classes: There is a link from node $\alpha$ to node $\beta$ labeled with action if the test represented by $\alpha$ is equivalent to (i.e., will always yield the same result as) executing action followed by test $\beta$. For instance, there is a link from the node representing the class containing $T$ ? (which we have indicated as $\overline{\text { CUR }}$ in Figure $3 \mathrm{e}$ ) to the node representing the class containing UT? (which we have indicated as $\overline{\mathrm{UP}}$ in Figure $3 \mathrm{e}$ ), and this link is labeled $D$ because executing the action $D$ followed by the test UT? is equivalent to executing the test $T$ ? (the $U$ and $\mathrm{D}$ actions cancel).

The boolean variable associated with each node represents the truth value of the corresponding test given the current global state of the environment. If the value of each node is known prior to performing an action, the value of each node following the action is easily determined by propagating values along the links. Consider again the two nodes $\alpha$ and $\beta$ with a directed link from $\alpha$ to $\beta$ labeled by action. Because test $\alpha$ is equivalent to action followed by test $\beta$, the value of $\beta$ after performing action is simply the value of $\alpha$ prior to the action. 
Based on the semantics of nodes and links, it is clear why each node has exactly one incoming link for each action. If a node $\beta$ received projections from two nodes, $\alpha_{1}$ and $\alpha_{2}$ for some action, this would imply that the equivalence classes $\alpha_{1}$ and $\alpha_{2}$ both contained the test consisting of action followed by test $\beta$. If the two equivalence classes contain the same test, they must represent the same equivalence class, and hence will be collapsed together.

\section{Acknowledgments}

Our thanks to Liz Jessup, Clayton Lewis, Rob Schapire, Paul Smolensky, Rich Sutton, and Dave Touretzky for helpful discussions and comments. This work was supported by NSF Presidential Young Investigator award IRI-9058450, grant 90-21 from the James S. McDonnell Foundation, and DEC external research grant 1250 to the first author; grant 87-2-36 from the Sloan Foundation to Geoffrey Hinton; and the Air Force Office of Scientific Research, Bolling AFB, under Grant AFOSR-89-0526, and the National Science Foundation under Grant ECS-8912623 to Andrew Barto.

\section{Notes}

1. The disadvantage of the update graph is that in degenerate, completely unstructured environments, the size of the update graph can be exponentially larger than the size of the FSA.

2. A consequence of this substitution is that error should not be back propagated from time $t$ to output units at times $t-1, t-2$, etc. It is not sensible to adjust the response properties of output units at time, say, $t-1$ to achieve the correct response at time $t$ because their appropriate activation levels have already been established by the sensations at time $t-1$.

3. Keeping the original value of $\mathbf{x}(t-\tau)$ is a somewhat arbitrary choice. Consistency can be achieved by propagating any value of $\mathbf{x}(t-\tau)$ forward in time, and there is no strong reason for believing $\mathbf{x}(t-\tau)$ is the appropriate value. We thus suggest two alternative schemes, but have not yet tested them. First, we might select $\mathbf{x}(t-\tau)$ such that the new $\mathbf{x}(t-i), i=0 \ldots \tau-1$, are as close as possible to the old values. Second, we might select $\mathbf{x}(t-\tau)$ such that the output units produce as close to the correct values as possible. Both these schemes require the computation-intensive operation of finding a least squares solution to a set of linear equations.

4. The definitive connectionist light bulb joke (courtesy of Thomas Mastaglio):

Q: How many connectionist networks does it take to change a light bulb?

A: Only one, but it needs about 6,000 trials.

5. We thank Rob Schapire for providing us with the latest results from his work.

6. Just as connectionist simulations require a bit of voodoo in setting learning rates, the RS algorithm has its own set of adjustable parameters that influence performance. One of us (JB) experimented with the RS algorithm, and without expertise in parameter tweaking, was unable to obtain performance in the same range as the measures reported in Table 2.

7. This is not a simple matter due to the fact that $\mathbf{W}^{\prime}$ is composed of $\mathbf{Q}^{*}$ as well as $\mathbf{Q}$ and the $\mathbf{Q}^{*}$ gradient must be transformed into a $\mathbf{Q}$ gradient. Consequently, we constrained $\mathbf{Q}$ to be an orthogonal matrix. For orthogonal matrices, $\mathbf{Q}^{-1}=\mathbf{Q}^{\mathrm{T}}$, which trivializes the mapping from $\mathbf{Q}^{*}$ gradients to $\mathbf{Q}$ gradients.

8. In the $n$-room world, there is only one sensation-the state of the light; thus, each test ends by evaluating this sensation. In environments having multiple sensations, tests can end with different sensations. 


\section{References}

Cohn, D., Atlas, L., Ladner, R., Marks II, R., El-sharkawi, M., Aggoune, M., \& Park, D. (1990). Training connectionist networks with queries and selective sampling. In D.S. Touretzky (Ed.), Advances in neural information processing systems 2 (pp. 566-573). San Mateo, CA: Morgan Kaufmann.

Elman, J.L. (1988). Finding structure in time (CRL Technical Report 8801). La Jolla: University of California, San Diego, Center for Research in Language.

Mozer, M.C. (1989). A focused back-propagation algorithm for temporal pattern recognition. Complex Systems, 3, 349-381.

Rivest, R.L., \& Schapire, R.E. (1987). Diversity-based inference of finite automata. In Proceedings of the TwentyEighth Annual Symposizm on Foundations of Computer Science (pp. 78-87).

Rivest, R.L., \& Schapire, R.E. (1987). A new approach to unsupervised learning in deterministic environments. In P. Langley (Ed.), Proceedings of the Fourth International Workshop on Machine Learning (pp. 364-375).

Rumelhart, D.E., Hinton, G.E., \& Williams, R.J. (1986). Learning internal representations by error propagation. In D.E. Rumelhart \& J.L. McClelland (Eds.), Parallel distributed processing: Explorations in the microstructure of cognition. Volume I: Foundations (pp. 318-362). Cambridge, MA: MIT Press/Bradford Books.

Schapire, R.E. (1988). Diversity-based inference of finite automata. Unpublished master's thesis, Massachusetts Institute of Technology, Cambridge, MA.

Servan-Schreiber, D., Cleeremans, A., \& McClelland, J.L. (1988). Encoding sequential structure in simple recurrent networks (Technical Report CMU-CS-88-183). Pittsburgh, PA: Carnegie-Mellon University, Department of Computer Science. 